\title{
Radiofrequency Catheter Ablation of Premature Ventricular Contractions from the Mitral Annulus in Patients without Structural Heart Disease
}

\author{
Yoshibumi Antoku ${ }^{1}$, Masao Takemoto ${ }^{2}$, Atsushi Tanaka ${ }^{3}$, Akihiro Masumoto ${ }^{4}$, Takahiro \\ Mito $^{5}$, Takafumi Ueno ${ }^{4}$, and Takuya Tsuchihashi ${ }^{1}$ \\ ${ }^{1}$ Social Medical Corporation Steel Memorial Yawata Hospital \\ ${ }^{2}$ Kyushu University Hospital \\ ${ }^{3}$ Saiseikai Fukuoka General Hospital \\ ${ }^{4}$ Fukuoka Kinen Hospital \\ ${ }^{5}$ Hakujuji Hospital
}

May 11, 2020

\begin{abstract}
INTRODUCTION: We previously reported the clinical benefits of radiofrequency catheter ablation (RFCA) of premature ventricular contractions (PVCs) from the right ventricular outflow tract or near His-bundle, which often can deteriorate clinical status and cause left ventricular (LV) dilation that develops into heart failure. This study aimed to evaluate the role of ablating PVCs per RFCA from a trans-interatrial septal approach in the LV dilation and clinical status in patients with PVCs from the mitral annulus (MA) (MA-PVCs) without structural heart disease. METHODS: The frequency of PVCs per the total heart beats by 24-h Holter monitoring, left ventricular ejection fraction (LVEF) and left ventricular end-diastolic (LVDd) and -systolic (LVDs) internal dimensions by echocardiography, New York Heart Association (NYHA) functional class, and serum brain natriuretic peptide (BNP) concentration in 22 patients with MA-PVCs were evaluated before and 6 months after RFCA. RESULTS: Before the RFCA, the patients with frequent $(20 \%<)$ PVCs demonstrated a significantly reduced LVEF, enlarged LVDd and LVDs, deteriorated NYHA functional class, and elevated serum BNP concentration as compared to the subgroup with rare ([?] 20\%) PVCs. Furthermore, ablating MA-PVCs readily produced an improvement in those abnormalities as compared to that before the RFCA. Interestingly, the MA-PVCs were successfully ablated from not only the ventricular side of the MA $(68 \%)$ or coronary sinus (5\%), but also the left atrial side of the MA (18\%). CONCLUSIONS: RFCA produces clinical benefits in patients with MA-PVCs. Further, it may be necessary to initially consider a trans-interatrial septal approach to ablate these PVCs.
\end{abstract}

\section{Condensed Abstract}

A subgroup of patients with frequent $(20 \%<)$ premature ventricular contractions (PVCs) from the mitral annulus (MA) (MA-PVCs) that significantly caused left ventricular (LV) dilation and deterioration of the clinical status as compared to that in the subgroup with rare ([?] 20\%) MA-PVCs. Furthermore, the RFCA of these arrhythmias was associated with a normalization of those abnormalities without any adverse effects. In addition, interestingly, because the MA-PVCs were successfully eliminated by radiofrequency catheter ablation (RFCA) from not only the ventricular side of the MA or coronary sinus but also the left atrial side of the MA, it may be necessary to initially consider a trans-interatrial septal approach to ablate these PVCs.

\section{Abbreviations and Acronyms}

$\mathrm{BNP}=$ brain natriuretic peptide 


$$
\begin{aligned}
& \text { LVDd }=\text { left ventricular end-diastolic internal dimension } \\
& \text { LVDs = left ventricular end-systolic internal dimension } \\
& \text { LVEF = left ventricular ejection fraction } \\
& \text { MA = mitral annulus } \\
& \text { MR = mitral regurgitation } \\
& \text { NYHA = New York Heart Association } \\
& \text { PDI = peak deflection index } \\
& \text { PVC = premature ventricular complexes } \\
& \text { RFCA = radiofrequency catheter ablation } \\
& \text { RVOT = right ventricular outflow tract } \\
& \% \text { PVC = frequency of premature ventricular complexes }
\end{aligned}
$$

\section{Introduction}

Isolated premature ventricular complexes (PVCs) are the most common arrhythmias observed in daily clinical practice in even patients without structural heart disease ${ }^{1}$. Many patients with PVCs often experience disabling symptoms and sometimes need long-term antiarrhythmic medications. In recent years, radiofrequency catheter ablation (RFCA) has proven to be a safe and successful therapy for arrhythmias including $\mathrm{PVCs}^{1,2}$. Moreover, we previously and recently reported the clinical benefit of RFCA of PVCs from the right ventricular outflow tract (RVOT-PVCs) ${ }^{1}$, which are one of the most common types of PVCs, and from near His-bundle ${ }^{2}$, which are a comparably uncommon type of PVC. In particular, eliminating frequent RVOT-PVCs by RFCA could steadily reverse RVOT-PVC induced left ventricular (LV) dilation, which is known to be a risk factor for LV dysfunction and congestive heart failure ${ }^{3}$. Although the PVCs from near the mitral annulus (MA) (MA-PVCs) are also comparably uncommon, they also often cause a deterioration of the patients' clinical status. Further, it is still unknown whether MA-PVCs cause LA dilation and / or dysfunction. Thus, the purpose of this study was two-fold and was 1) to examine whether frequent MAPVCs may cause LV dilation and / or dysfunction and 2) to evaluate the effect of ablating PVCs by RFCA from a trans-interatrial septal approach on the LV dilation and clinical status in symptomatic patients with frequent MA-PVCs without structural heart disease.

\section{Material and Methods}

Study population and laboratory analysis : This study was approved by the institutional review committee and ethics review board of our hospitals. From 2014 to 2019, 22 consecutive intolerably symptomatic patients with drug (including beta-blockers, calcium-channel blockers, and class I agents of the Vaughan Williams classification) refractory MA-PVCs visited our hospitals to undergo RFCA. All patients had their history recorded, and underwent a physical examination, laboratory analysis, 12-lead electrocardiography (12-ECG), 24-h Holter monitoring, and echocardiography, on admission or within at least 1 month before admission, and 6-months after the successful RFCA. The LV end-diastolic (LVDd) and systolic (LVDs) internal dimensions, right ventricle (RV) internal dimension (RVD), degree of mitral regurgitation (MR), and LV ejection fraction (LVEF) measured by the Teichholz method, were measured. All the echocardiography values were recorded during sinus rhythm, but not during PVC beats, nor post-PVC beats. The serum brain natriuretic peptide (BNP) concentration and New York Heart Association (NYHA) functional class using a specific activity scale were evaluated on admission and 6-months after the successful RFCA.

Definition of MA-PVC: Mitral annulus-PVCs were defined as having a characteristic electrocardiographic appearance of a right bundle branch block (RBBB) contour in $\mathrm{V} 1$ and positive concordant $\mathrm{R}$ waves in the precordial leads including V1 6 (Figure 1A). Ventricular tachycardia (VT) was defined with standard electrocardiographic criteria including at least 3 consecutive PVCs at a rate of $>120$ beats per minute. 
Patients with ventricular tachycardia and atrial tachyarrhythmias including atrial fibrillation, atrial flutter, atrial tachycardia, and paroxysmal supraventricular tachycardia were excluded from this study because they may cause tachycardia-induced LV dilation ${ }^{4}$. The patients who received hemodialysis during this study were also excluded from this study.

Mapping and catheter ablation procedure: All procedures were performed after written informed consent was obtained. Anti-arrhythmic drugs were withdrawn in all patients at least five half-lives before the procedure. The anti-hypertensive and -hypercholesterolemic agents and/or others were continued in patients with hypertension and dyslipidemia before and after the RFCA. The anti-diabetic agents in patients with diabetes mellitus were continued before and after the RFCA but not on the procedural day. The QRS duration and peak deflection index (PDI $)^{5}$ were measured before the RFCA. RFCA was performed under local anesthesia. After a 50 unit per kilogram administration of heparin was administered, 4 or 5 -mmtip electrode catheters (St. Jude Medical, St. Paul, MN, USA) were introduced percutaneously into the right ventricle (RV) and coronary sinus, respectively. A 100 unit per kilogram administration of heparin was administered following the trans-interatrial septal puncture, and heparinized saline was additionally infused to maintain the activated clotting time at 300-350 seconds. After left atrio- and ventriculography, electro-anatomical mapping during the PVCs was performed in the coronary sinus and around the MA, left atrium (LA), and LV near the MA in detail utilizing a 3D mapping system (EnSite NavX / Velocity ${ }^{\text {TM }}$ Cardiac Mapping System, St. Jude Medical, St. Paul, MN, USA) by a circular mapping catheter (Inquiry ${ }^{\text {TM }}$ AFocusII ${ }^{\mathrm{TM}}$ Double Loop Catheter, St. Jude Medical, St. Paul, MN, USA) or high density mapping catheter (Advisor ${ }^{\mathrm{TM}}$ HD Grid catheter, Abbott, Plymouth, Minn, USA) during culprit PVCs. If the culprit PVC was not found during the procedure, an isoproterenol administration and/or programmed electrical stimulation with a digital stimulator (Cardiac Stimulator, Nihon Kohden Co., Tokyo, Japan) was performed to induce the culprit PVC. Then, an open irrigated 3.5-mm-tip ablation catheter (FlexAbility ${ }^{\mathrm{TM}}$, St. Jude Medical, St. Paul, MN, USA) through a steerable introducer (Agilis ${ }^{T M}$ NxT, Abbott, Plymouth, Minn, USA) was positioned at the MA. An optimal pace map was defined as a match of all 12 surface leads by comparing the $\mathrm{R} / \mathrm{S}$ ratio and subtle notching in the QRS complex during pacing. An identical match was necessary in at least 11 of 12 leads. Further, based on the findings of the electro-anatomical mapping and an optimal pace map, radiofrequency energy was delivered at the site of the unipolar potentials from the ablation catheter demonstrating a QS pattern and bipolar potentials from the tip of the ablation catheter preceding the QRS in the 12-ECG by at least 20 -30 milliseconds during the PVC with a preset temperature of 43 to $50^{\circ} \mathrm{C}$ and power limit of 30-35 W. Further, when the radiofrequency energy was delivered from the coronary sinus, in order to avoid any complications, (1) heparinized saline was additionally infused to maintain the activated clotting time at more than $300 \mathrm{~s}$, (2) a maximum power setting of $20 \mathrm{~W}$ for the radiofrequency energy was chosen, and (3) careful attention to and observation of the patient was paid during the radiofrequency energy delivery as we previously reported ${ }^{6}$. The radiofrequency energy application was terminated when an abnormal impedance rise $(>30 \Omega)$ was observed. A successful ablation was defined as no recurrence and non-inducibility of the culprit PVC with and without an isoproterenol administration and/or programmed electrical stimulation for at least 30 minutes after the ablation. Procedural success was defined as no recurrence of the culprit PVC within 24 hours after the procedure under electrocardiogram monitoring. If the recurrence of the culprit PVC was observed, a repeat RFCA was performed.

Statistical Analysis: The numerical results are expressed in the text as the mean \pm standard deviation. Paired data was compared by a Fisher's exact test and Student $t$ tests. The differences between the continuous variables before and 6 months after the RFCA in each group, or between the lower and upper groups in each period, were compared by using a repeated measures analysis of variance followed by a Bonferroni's test for multiple comparisons. The correlation between the two parameters was determined by a simple linear regression analysis. All analyses were performed with SAS version 9.2 software (SAS Institute, Cary, NC). A p of $<0.05$ was considered to indicate statistical significance.

\section{Results}

Clinical Characteristics of the Patients with MA-PVCs on Admission: The frequency of PVCs 
(\%PVC) was calculated as: [number of PVC / number of total heart beats per 24 hours] x 100. The mean \%PVC of the 22 patients (14 males and 8 females with a mean age of $61 \pm 19$ years, body mass index [BMI] of $25.3 \pm 4.9 \mathrm{~kg} / \mathrm{m}^{2}$, and serum creatinine of $0.95 \pm 0.78 \mathrm{mg} / \mathrm{dl}$ ) in this study was $20.1 \pm 8.6 \%$. The relationships between the LVDd and \%PVC by 24-h Holter monitoring in the 22 patients before the RFCA were examined (Figure 2A). A significant correlation was found between the LVDd and \%PVC $(\mathrm{y}=29.43+$ $15.76 \mathrm{x}, \mathrm{r}=0.499, \mathrm{p}=0.018)$, indicating that frequent PVCs might cause LV dilation. Thus, the 22 patients were divided into two groups (the lower [[?] 20\%; $\mathrm{n}=14]$ and upper [20\% $<; \mathrm{n}=8]$ groups) according to the quantile of the \%PVC. The baseline characteristics of the patients are shown in Table 1 . There were no statistical differences among all the factors except no medication including the age $(55+-19$ versus 71 +- $14 ; \mathrm{p}=0.062)$, BMI $\left(24.9+-4.7\right.$ versus $\left.26.1+-5.4 \mathrm{~kg} / \mathrm{m}^{2} ; \mathrm{p}=0.607\right)$, serum creatinine $(0.76+-0.12$ versus $1.30+-1.27 ; \mathrm{p}=0.121)$, prevalence of an inferior $(93 \%$ versus $100 \% ; \mathrm{p}=0.463)$ or superior axis $(7 \%$ versus $0 \% ; \mathrm{p}=0.174)$ in the frontal plane of the 12 -ECG of the MA-PVCs, being male (64\% versus $63 \% ; \mathrm{p}=0.683)$, co-existence of hypertension ( $36 \%$ versus $75 \% ; \mathrm{p}=0.083)$, dyslipidemia $(21 \%$ versus $38 \%$; $\mathrm{p}=0.440)$, diabetes mellitus $(14 \%$ versus $25 \% ; \mathrm{p}=0.553)$, ex- or current smoking $(50 \%$ versus $38 \% ; \mathrm{p}=$ $0.592)$, prevalence of symptoms including palpitations $(93 \%$ versus $100 \% ; \mathrm{p}=0.463)$, general fatigue $(43 \%$ versus $50 \% ; \mathrm{p}=0.760)$, chest discomfort / pain $(21 \%$ versus $50 \% ; \mathrm{p}=0.182)$, fainting $(14 \%$ versus $13 \% ; \mathrm{p}=$ 0.912 ), and medications including beta-blockers ( $43 \%$ versus $75 \% ; \mathrm{p}=0.160)$, calcium channel blockers $(21 \%$ versus $13 \% ; \mathrm{p}=0.622)$, and class I agents $(21 \%$ versus $35 \% ; \mathrm{p}=0.856)$ between the Lower and Higher group. The prevalence of no medications $(43 \%$ versus $0 \% ; \mathrm{p}=0.030)$ in the Lower group was higher than that in the Higher group. No patients were on amiodarone or sotalol. Although those agents were not sufficiently effective in eliminating the PVC-associated symptoms before the RFCA, all of the patients with a successful procedure reported the absence of any PVC-associated symptoms and could discontinue the antiarrhythmic agents after the RFCA. The mean age of the patient, serum creatinine, and prevalence of hypertension were slightly older and higher in the Higher group than Lower group, but the differences were not significant between the two groups.

Analysis of the 24-h Holter monitoring, New York Heart Association (NYHA) functional class, and serum brain natriuretic peptide (BNP) concentration: Table 2 summarizes the analysis of the 24-h Holter monitoring, NYHA functional class by the specific activity scale, and serum BNP concentration. In 20 patients who achieved procedural success, the number of total heart beats did not statistically differ before $(106,911+-13,950$ versus $110,788+-9,666$ beats per day; $\mathrm{p}=0.504)$ and after $(106,506+-12,689$ versus 106,604 +- 7,623 beats per day; $\mathrm{p}=0.985)$ the RFCA between the Lower and Higher groups. The RFCA significantly reduced the number of total PVCs $(16,046+-3,804$ versus $54+-31$ beats per day; $\mathrm{p}<$ 0.001 and $32,927+-10,439$ versus $147+-161$ beats per day; $\mathrm{p}<0.001)$ and \%PVC $(14.9+-2.3$ versus 0.05 +- $0.03 \% ; \mathrm{p}<0.001$ and $29.3+-7.6$ versus $0.14+-0.14 \% ; \mathrm{p}<0.001)$ with no evidence of a recurrence of the culprit PVC in the Lower and Higher groups. There was no statistical difference in the number of total PVCs $(54+-31$ versus $147+-161$ beats per day; $\mathrm{p}=0.065)$ and \%PVC $(0.05+-0.03$ versus $0.14+-0.14 \%$; $\mathrm{p}=0.064)$ after the RFCA between the two groups. There was no statistical difference in the frequency of premature atrial complexes before and after the RFCA between the three groups (data not shown). The NYHA functional class and serum BNP concentration were evaluated in all patients on admission for the RFCA. Before the RFCA, the NYHA functional class and serum BNP concentration were demonstrated to be significantly worse and higher in the Higher group than in the Lower group $(1.67+-0.49$ versus 2.50 $+-0.53 ; \mathrm{p}=0.002$ and $44+-28$ versus $145+-94 ; \mathrm{p}=0.003)$. RFCA significantly improved the NYHA functional class and serum BNP concentration in both the Lower $(1.67+-0.49$ versus $1.00+-0.00 ; \mathrm{p}<$ 0.001 and $44+-28$ versus $20+-12 ; \mathrm{p}=0.014)$ and Higher $(2.50+-0.53$ versus $1.00+-0.00 ; \mathrm{p}<0.001$ and $145+-94$ versus $40+-26 ; \mathrm{p}=0.015)$ groups as compared to that before the intervention.

Analysis of the Echocardiogram: Figure 2 summarizes the results of the analysis of the echocardiograms. Before the RFCA, a subgroup of patients in the Higher group demonstrated a significantly reduced LVEF $(73+-8$ versus $61+-8 \% ; \mathrm{p}=0.005)$, enlarged LVDd $(48+-5$ versus $53+-5 \mathrm{~mm} ; \mathrm{p}=0.042)$ and LVDs $(31+-5$ versus $38+-4 \mathrm{~mm} ; \mathrm{p}=0.002)$, as compared to the Lower groups (Figure 2BCD). However, at the 6 month examination after the RFCA, all of those abnormalities seen in the Higher group before the 
RCFA had reversed (Figure 2BCD), and there was no statistical difference in these parameters between the two groups. There was no statistically differences in the RVD and MR before and after the RFCA between the two groups (Figure 2EF).

Electrophysiological Findings and Approach Sites with a Successful RFCA: Table 3 summarizes the results of the electrophysiological findings and successful ablation sites. An RFCA procedure for MAPVCs was performed in 22 patients. Procedural success was achieved in 20 (91\%) of 22 patients. No patients suffered from any procedure-related complications. During the follow-up, recurrence of the culprit MA-PVCs was observed in one patient (5\%), who underwent a repeat RFCA with a successful result. The mean QRS duration and PDI of the PVCs in the 22 patients were $142+-28 \mathrm{~ms}$ and $0.60+-0.07$, respectively. There were no statistical differences in the QRS duration $(145+-27$ versus $135+-29 \mathrm{~ms} ; \mathrm{p}=0.235)$ and PDI $(0.61+-0.08$ versus $0.57+-0.06 ; \mathrm{p}=0.438)$ of the PVCs between the Lower and Higher groups.

The approach sites of the MA-PVCs for the RFCA are demonstrated in Figure 3B-F. Of the 22 patients, in $13(59 \%), 2(9 \%)$, and $1(5 \%)$ patient a successful RFCA on the ventricular side of the MA from the supra-mitral valve (Figure 3B) and infra-mitral valve (Figure 3C) using the trans-interatrial septal approach and trans-coronary sinus approach (Figure 3E) was achieved. Interestingly, in $4(18 \%)$ patients a successful RFCA on the LA side of the MA using a trans-interatrial septal approach was achieved (Figure 3D). A successful RFCA of the MA-PVCs could be successful RFCA of MA-PVCs in 20 patients with these steps. The notable point is that, of the 20 patients with a successful RFCA, in $19(95 \%)$ patients a successful RFCA using a trans-interatrial septal approach could be achieved. However, in the remaining 2 patients successful results could not be achieved even though additional RFCA using the trans-aortic approach was performed (Figure 3F).

Figure 1B-G demonstrated that the EnSite ${ }^{\mathrm{TM}}$ 3D-mapping images of the earliest activation sites during the culprit PVC (Figure 1BE) and intra-cardiac electrocardiograms (Figure 1CF) and 12-ECGs of the pace map (Figure 1DG) at the successful ablation sites. The EnSite ${ }^{\mathrm{TM}}$ 3D-mapping images demonstrated that the earliest activation sites during the culprit PVCs were confirmed in the white area on the ventricular (Figure 1B) and LA (Figure 1E) side of the MA. The yellow points were the successful ablation sites. The findings from the unipolar potentials recorded by the ablation catheter demonstrated a QS pattern, bipolar potentials were recorded by the tip of the ablation catheter that proceeded the QRS in the 12-ECG by 32 ms (Figure 1C) during the culprit PVC, and a suboptimal pace map during pace mapping at 9.9 V (Figure 1D) was obtained by the ablation catheter placed at the successful ablation site on the ventricular side of the MA using a supra-mitral valve approach (Figure 3B). Of course, the atrial potentials were not confirmed by the tip of the ablation catheter. On the other hand, the findings from the unipolar potentials recorded by the ablation catheter demonstrated a QS pattern and bipolar potentials recorded by the tip of the ablation catheter that proceeded the QRS in the 12-ECG by $48 \mathrm{~ms}$ (Figure $1 \mathrm{~F}$ ) during the culprit PVC were obtained by the ablation catheter placed at successful ablation site on the LA side of the MA (Figure 1E). Of course, the atrial potential was confirmed by the tip of the ablation catheter, and pace mapping at $9.9 \mathrm{~V}$ in the LA side of MA (Figure 1E) captured the LA but not the LV (Figure 1G). After radiofrequency energy was delivered at those points, the PVCs were steadily terminated.

Figure 3G summarizes the earliest activation sites of the MA-PVCs that were successful (red circle; $\mathrm{n}=$ 20 ) or unsuccessful (black circle; $n=2$ ) RFCA sites in the left anterior oblique view of the MA. They were crowded in the direction from 0:00 to 3:00 in the left anterior oblique view of the MA.

Outcome of patients with an unsuccessful RFCA. Of the 22 patients, in 2 patients in the Lower group, unfortunately the procedure was unsuccessful during this study. One patient received a repeat RFCA with a successful result. Because the other did not want to receive a repeat RFCA, medical therapies were continued. Tables 1, 2, and 3 and Figure 2A-D summarizes the clinical characteristics (Table 1), results of the analysis of the 24-h Holter monitoring, NYHA functional class, serum BNP concentration (Table 2), echocardiograms (Figure 2B-F), and electrophysiological findings (Table 3) of those 2 patients who looked like men with a slender build (Table 1). All of those factors including the \%PVC, NYHA functional class, serum BNP concentration, and LVDd, LVDs, and LVEF obtained by the echocardiogram, in the 2 patients 
with an unsuccessful RFCA still deteriorated further during the follow-up. The mean QRS duration and PDI were $153 \mathrm{~ms}$ and 0.57 , respectively.

\section{Discussion}

Clinical Characteristics in Patients with PVCs from the MA: We demonstrated that although the differences were not significant between the two groups, the mean patient age, serum creatinine, and prevalence of hypertension were slightly older and higher in the Higher group than Lower group. Hypertension causing a pressure overload of the LV and aging contributing to myocardial degeneration may have affected the increase in the MA-PVCs. The prevalence of no medication $(43 \%$ versus $0 \% ; \mathrm{p}=0.030)$ in the Lower group was higher than that in the Higher group. The family doctors may have tended to prescribe antiarrhythmic agents to the patients with a higher frequency of PVCs.

Clinical Benefits of RFCA of MA-PVCs: This retrospective study provided evidence in support of the concept that some patients with frequent MA-PVCs of more than $20 \%$ of the total heart beats, without any overt underlying structural heart disease, were associated with LV dilation, which is known to be a risk factor for LV dysfunction and congestive heart failure ${ }^{3}$. Before the RFCA, the parameters including the LVEF, LVDd, LVDs, NYHA functional class, and serum BNP concentration, in those higher PVC-patients (20\% $<$ ) were worse than in the lower PVC-patients ([?] 20\%) (Figure 2ABCD, Table 2). Those abnormalities were still present one day after a successful RFCA. Furthermore, those parameters in the patient with an unsuccessful RFCA still deteriorated further during the follow-up. Those findings may have indicated that the frequent MA-PVCs of more than $20 \%$ of the total heart beats, may be an important and independent risk factor for the progression of LV dysfunction and heart failure. The findings of an elevated serum BNP concentration (Table 2) before the RFCA may also support the possibility of heart failure progression. More importantly, ablating the MA-PVCs readily produced an improvement in those abnormalities without any adverse effects. Attenuation of the progression of the LV dilation has been proven to reduce the risk of cardiovascular events ${ }^{3}$, and RFCA is known to be safe and effective for the treatment of arrhythmias as compared to medical therapy ${ }^{1,2}$. Thus, RFCA may be considered as the first choice of therapy in patients with frequent MA-PVCs.

RFCA of MA-PVCs : A recent report demonstrated that in 18 of 21 patients (86\%) a successful ablation could be achieved in those with MA-PVCs/VT $\mathrm{VT}^{7}$. Procedural success was achieved in $20(91 \%)$ of the 22 patients, which was slightly higher than that of the previous report ${ }^{7}$. In order to achieve a high procedural successful rate, we tried and paid attention to the 3 points described below. (1) First, to gain a strong support and contact force of the ablation catheter, the trans-interatrial septal approach was initially performed and a steerable introducer (Agilis ${ }^{\mathrm{TM}} \mathrm{NxT}$, Abbott, Plymouth, Minn, USA) was routinely used. Actually, of those 20 patients with a successful procedure, in 19 (95\%) a successful RFCA of the MA-PVCs could be achieved with the trans-interatrial septal approach on the ventricular side of the MA from the supra-mitral valve $(59 \%)$ (Figure 3B) and infra-mitral valve (9\%) (Figure 3C) and on the LA side of the MA (18\%) (Figure 3D) (Table 3). Interestingly, in 4 patients (18\%) a successful RFCA was achieved on the LA side of the MA using the trans-interatrial septal approach (Figure 3D). Thus, operators should try to ablate MA-PVCs on the LA side of the MA (Figure 3D), when those PVCs cannot be ablate on the ventricular side of the MA (Figure 3BC). It may be hard for the ablation catheter to reach the LA using the transaortic approach (Figure 3E). Moreover, in the remaining 2 patients (9\%) with an unsuccessful procedure, successful results could not be achieved even though a trans-aortic approach was additionally performed ${ }^{8}$ (Figure 3F). The trans-aortic approach was not considered to be able to gain a stronger support and contact force than the trans-interatrial septal approach. Further, the remaining 1 patient (5\%) underwent a successful procedure that could be achieved from a coronary sinus approach ${ }^{6,8}$ Thus, a right-sided approach including a trans-interatrial septal and coronary sinus approach may be considered first, when MA-PVCs need to be ablated. (2) Second, to detect more precise earliest activation sites of culprit MA-PVCs, electro-anatomical mapping was performed in detail using a circular mapping catheter (Inquiry ${ }^{\mathrm{TM}}$ AFocusII $^{\mathrm{TM}}$ Double Loop Catheter, St. Jude Medical, St. Paul, MN, USA) or high density mapping catheter (Advisor ${ }^{\mathrm{TM}}$ HD Grid catheter, Abbott, Plymouth, Minn, USA) on the both LV and LA side of MA. (3) Third, to obtain a stable 
lesion creation, an open irrigated 3.5-mm-tip ablation catheter (FlexAbility ${ }^{\mathrm{TM}}$, St. Jude Medical, St. Paul, MN, USA) was routinely used. However, unfortunately, in 2 patients (5\%) successful results could not be achieved even though RFCA using open irrigated ablation catheters from the various approach sites described in Figure 3B-F was performed. The recent advancements in the new technologies, including contact force catheters $^{9}$, which can facilitated a more stable lesion creation, were not used in this study, and they might have improved and helped during the RFCA procedure in those unsuccessful patients using open irrigated ablation catheters.

Distribution of the Earliest Activation Sites of the MA-PVCs : The earliest activation sites of the MA-PVCs were crowded in the direction from 0:00 to 3:00 in the left anterior oblique view of the MA (Figure 3G) where the left atrial appendage was attached. Although there has been a case report supporting a possible association between ventricular arrhythmias and the LA appendage ${ }^{10}$, the detailed mechanism (s) of MA-PVCs still remain unidentified.

Treatment of patients with an unsuccessful RFCA. Of the 22 patients, in 2 in the Lower group the procedure was unsuccessful during this study. All of the parameters including the \%PVC, NYHA functional class, serum BNP concentration, and LVDd, LVDs, and LVEF obtained by the echocardiogram, in the 2 patients with an unsuccessful RFCA still deteriorated further during the follow-up (Table 1-3, Figure 2B-F). Thus, intensive treatment of heart failure including the administration of renin-angiotensin system inhibitors and diuretics was started after the unsuccessful RFCA, resulting in a comparative improvement in the patient symptoms. The class I agents were discontinued because of the adverse effects of the long-term antiarrhythmic medications associated with those agents such as an increased mortality, probably due to their proarrhythmic effects, even though the occurrence of the PVCs was markedly suppressed ${ }^{11}$.

Comparison of the PVCs from the MA, RVOT ${ }^{1}$, and near the His-bundle ${ }^{2}$ : This study has demonstrated that MA-PVCs were common in males in their 60s, and the QRS duration was wide (142 +$28 \mathrm{~ms}$ ) and caused LV dilation. We previously showed that RVOT-PVCs ${ }^{1}$ were common in females in their $50 \mathrm{~s}$, and the QRS duration was wide $(141+-25 \mathrm{~ms})$ and caused LV dilation, and PVCs from near the Hisbundle (His-PVCs) ${ }^{2}$ were common in males in their 70s, and the QRS duration was narrow (119+- $\left.23 \mathrm{~ms}\right)$ and did not cause LV dilation. We also previously reported that frequent RVOT-PVCs $(20 \%<)$ increased $\mathrm{MR}$, and eliminating those PVCs produces an improvement in the $\mathrm{MR}^{1}$, but not the frequent MA-PVCs (20\%) in this study. RVOT-PVCs cause left branch bundle block (LBBB), which causes a dyssynchronous myocardial activation, which progresses into $\mathrm{MR}^{12}$. On the other hand, the MA-PVCs caused RBBB, which may not have a great enough effect on the dyssynchronous myocardial activation. Thus, although the clinical background differed in the patients with those MA-, RVOT, and His-PVCs, frequent $(20 \%<)$ PVCs with a wider QRS duration may be one of the important predictors of LV dilation.

\section{Limitations of the Study}

Although our study was a multi-center trial, it was limited by a retrospective design and relatively small number of patients because MA-PVCs are comparatively rare arrhythmias. The patients in this study were also limited by having a high frequency of \%PVCs (mean \% PVC $=20.1+-8.6 \%$ ) and disabling symptoms. Thus, the effect of the RFCA of the MA-PVCs in patients with a low frequency of \%PVCs and/or asymptomatic patients was unfortunately unknown in this study. Moreover, our study could not clarify the long term clinical benefit of RFCA of MA-PVCs. Thus, whether our results can safely be extrapolated to the inclusion of a larger number of patients, whether the use of contact force catheters, and whether a longer follow-up period in these patients is needed should be determined in further studies.

\section{Conclusions}

A subgroup of patients with frequent $(20 \%<)$ MA-PVCs demonstrated a significantly enlarged LVDd and LVDs, reduced LVEF, deteriorated NYHA functional class, and elevated serum BNP concentration as compared to the subgroup with rare ([?] 20\%) MA-PVCs. Furthermore, the RFCA of these arrhythmias was associated with the normalization of these abnormalities without any adverse effects. These findings suggest thatsimilar to RVOT-PVCs, MA-PVCs may be a possible cause of LV dysfunction and/or heart failure, as 
previously reported ${ }^{1}$. Thus, the physicians should be aware of this condition when examining patients with LV dilation and/or a reduced LVEF in the presence of frequent MA-PVCs because it may be at least one important risk factor for the progression of LV dysfunction and heart failure.

In addition, initially performing a trans-interatrial septal approach and using a steerable introducer to gain a stronger support and contact force of the ablation catheter, performing detailed electro-anatomical mapping using by a circular mapping catheter or high density mapping catheter to detect more precise earliest activation sites of the target MA-PVCs, and using open irrigated ablation catheters to obtain a stable lesion creation, may be one of the important procedures to achieve a high successful rate of the RFCA of MA-PVCs. Interestingly, because a successful RFCA was achieved for the MA-PVCs from not only the ventricular side of the MA (Figure 3BC) or coronary sinus (Figure 3E) but also from the LA side of the MA (Figure 3D), it may be necessary to understand the cardiac anatomy and try to ablate those PVCs from various approach sites, especially using a trans-interatrial septal approach initially may be helpful. Because these PVCs are often drug-refractory, RFCA may be considered as the first choice of therapy in those patients.

\section{References}

1. Takemoto M, Yoshimura H, Ohba Y, et al. Radiofrequency catheter ablation of premature ventricular complexes from right ventricular outflow tract improves left ventricular dilation and clinical status in patients without structural heart disease. J Am Coll Cardiol. 2005;45:1259-1265

2. Tanaka A, Takemoto M, Masumoto A, et al. Radiofrequency catheter ablation of premature ventricular contractions from near the his-bundle. J Arrhythm. 2019;35:252-261

3. Vasan RS, Larson MG, Benjamin EJ, et al. Left ventricular dilatation and the risk of congestive heart failure in people without myocardial infarction. N Engl J Med. 1997;336:1350-1355

4. Shinbane JS, Wood MA, Jensen DN, et al. Tachycardia-induced cardiomyopathy: A review of animal models and clinical studies. J Am Coll Cardiol. 1997;29:709-715

5. Hachiya H, Hirao K, Sasaki T, et al. Novel ecg predictor of difficult cases of outflow tract ventricular tachycardia: Peak deflection index on an inferior lead. Circ J. 2010;74:256-261

6. Mito T, Takemoto M, Kang H, et al. A case of premature ventricular complexes/ventricular tachycardia from the left ventricular outflow tract successfully ablated from the distal great cardiac vein. J Cardiol Cases. 2017;16:85-88

7. Yue-Chun L, Cheng Z, Jun H, et al. Catheter ablation of idiopathic premature ventricular contractions and ventricular tachycardias originating from the vicinity of endocardial and epicardial mitral annulus. PLoS One. 2013;8:e80777

8. Lian-Pin W, Yue-Chun L, Jing-Lin Z, et al. Catheter ablation of idiopathic premature ventricular contractions and ventricular tachycardias originating from right ventricular septum. PLoS One. 2013;8:e67038

9. Ariyarathna N, Kumar S, Thomas SP, et al. Role of contact force sensing in catheter ablation of cardiac arrhythmias: Evolution or history repeating itself? JACC Clin Electrophysiol. 2018;4:707-723

10. Yakubov A, Salayev O, Hamrayev R, et al. A case of successful ablation of ventricular tachycardia focus in the left ventricular summit through the left atrial appendage: A case report. Eur Heart J Case Rep. 2018;2:yty110

11. Akhtar M, Breithardt G, Camm AJ, et al. Cast and beyond. Implications of the cardiac arrhythmia suppression trial. Task force of the working group on arrhythmias of the european society of cardiology. Circulation. 1990;81:1123-1127

12. Littmann L, Symanski JD. Hemodynamic implications of left bundle branch block. J Electrocardiol. 2000;33 Suppl:115-121

Figure legends 
Figure 1. The 12-lead electrocardiograms in the patients with premature ventricular contractions from the mitral annulus (MA) (A). The EnSite ${ }^{\mathrm{TM}}$ 3D-mapping images of the earliest activation sites during the culprit PVCs (BE), intra-cardiac electrocardiograms (CF), and 12-ECGs during the pace map (DG) at the successful ablation sites on the ventricular side of the MA (BCD) or left atrium side of the MA (EFG) with the trans-interatrial septal approach.

Figure 2. The scatter plots showing the relationship between the left ventricular end-diastolic internal dimension (LVDd) and frequency of premature ventricular complexes (\%PVC) $(\mathrm{n}=22)(\mathrm{A})$. The analysis of the left ventricular ejection fraction (LVEF) (B), left ventricular end-diastolic internal dimension (LVDd) (C), left ventricular end-systolic internal dimension (LVDs) (D), right ventricular internal dimension (RVD) (E), and degree of mitral regurgitation (MR) (F) by echocardiogram before (blue bars) and 6 months after (red bars) the radiofrequency catheter ablation (RCFA) in the Lower (\%PVC [?] 20\%), Higher $(20 \%<$ ), and unsuccessful RFCA groups. ${ }^{*} \mathrm{p}<0.05$ versus before and after the RFCA in each group; $+\mathrm{p}<0.05$ versus the Lower and Higher groups during each period.

Figure 3. The left atrio- and ventriculography (A) and the approach sites of the radiofrequency catheter ablation on the ventricular side of the mitral annulus (MA) from the supra-mitral valve (B), infra-mitral valve $(\mathrm{C})$, and left atrial side of the MA (D) from the trans-interatrial septal approach, and from the transcoronary sinus approach (E), and from the trans-aortic approach (F). The LA, LV, MV, ABL, CS, and His-RV, indicate the left atrium, left ventricle, mitral valve, ablation catheter, coronary sinus, and Hisbundle and right ventricle, respectively. The upper and middle, and lower panels were the right (RAO) and left anterior oblique (LAO) view of the LA and LV, respectively. The red, blue, and water blue bar indicate the ablation catheter and the electrodes placed on the CS and His-RV, respectively. The earliest activation sites of the premature ventricular contractions from the MA with a successful (red circle; $\mathrm{n}=20$ ) or unsuccessful (black circle; $\mathrm{n}=2$ ) radiofrequency catheter ablation in the LAO view of the MA.

\section{Figure 1A}

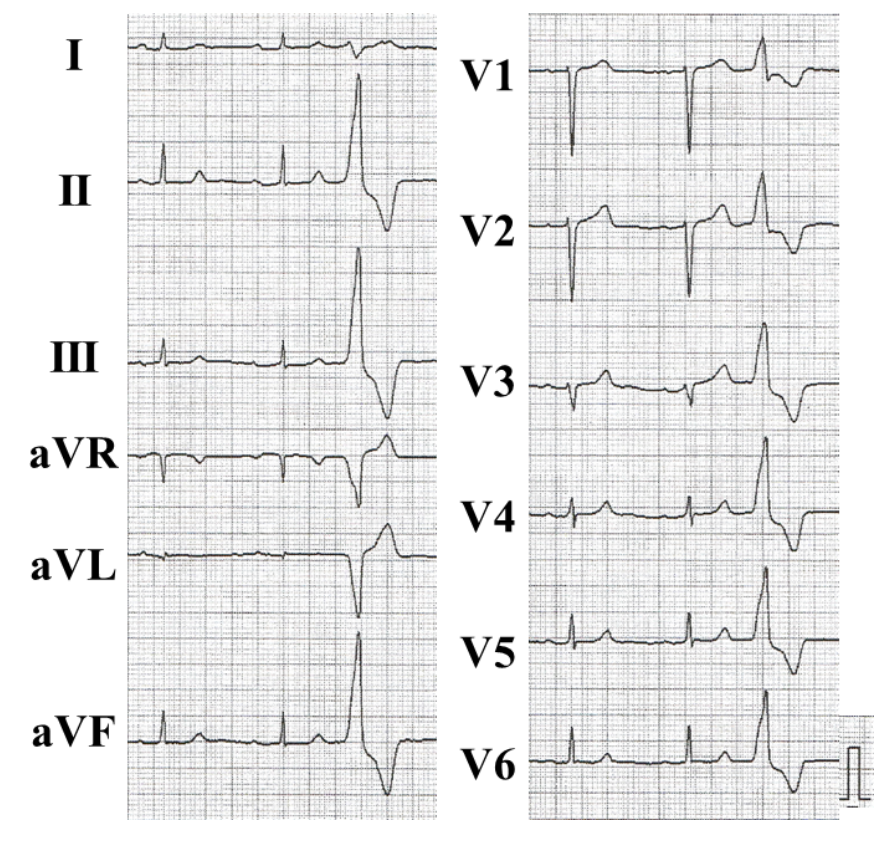



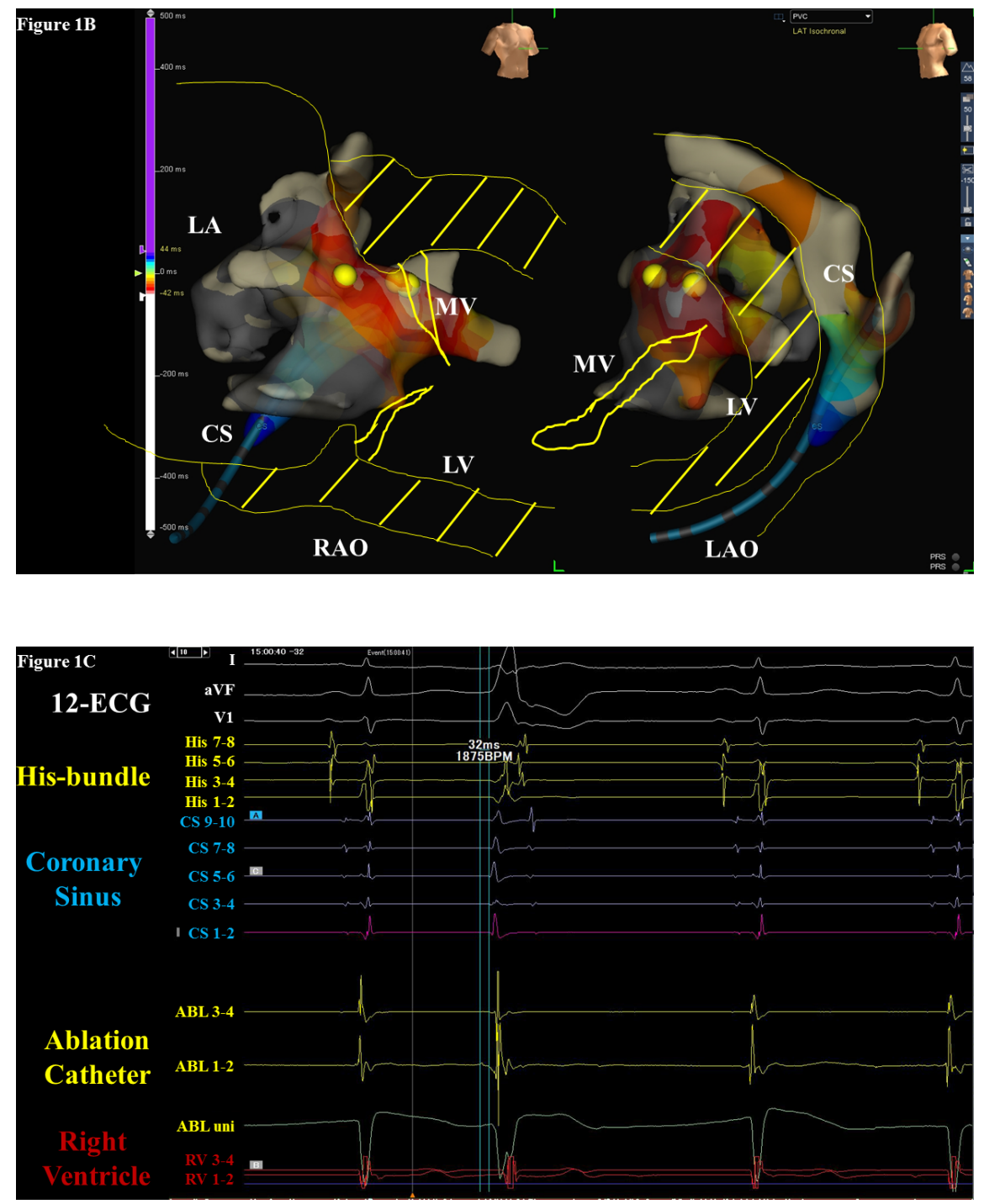

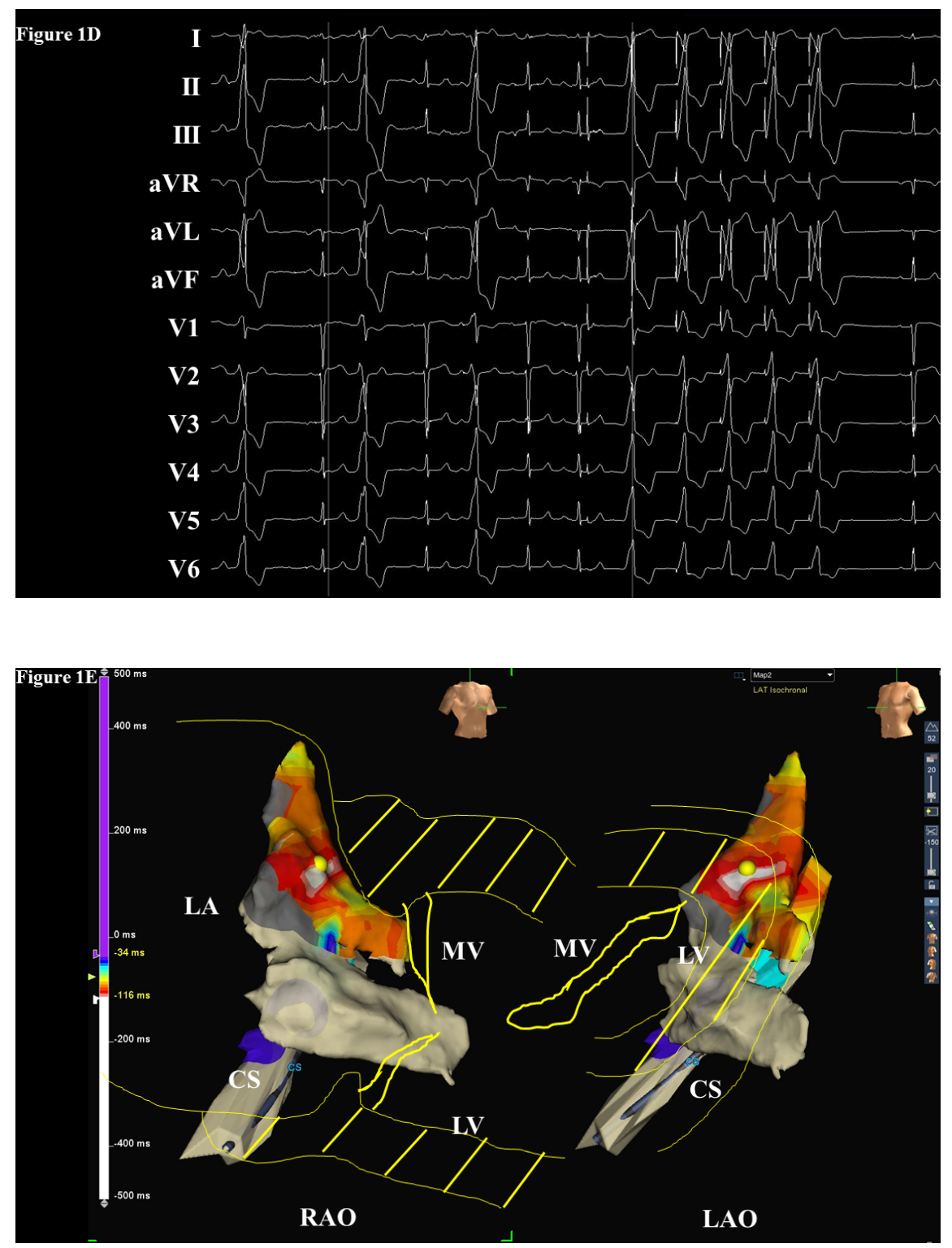

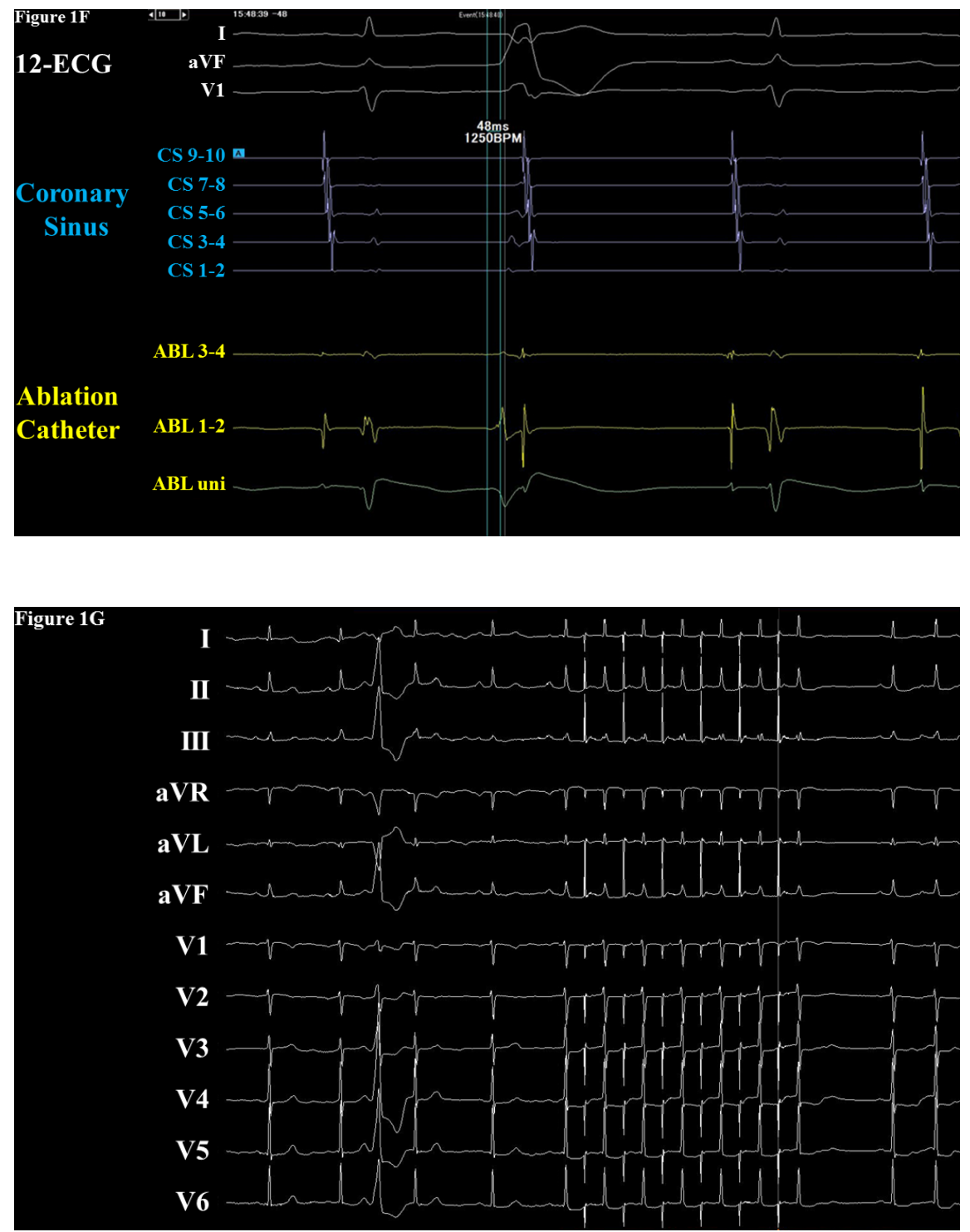

\section{Figure 2}
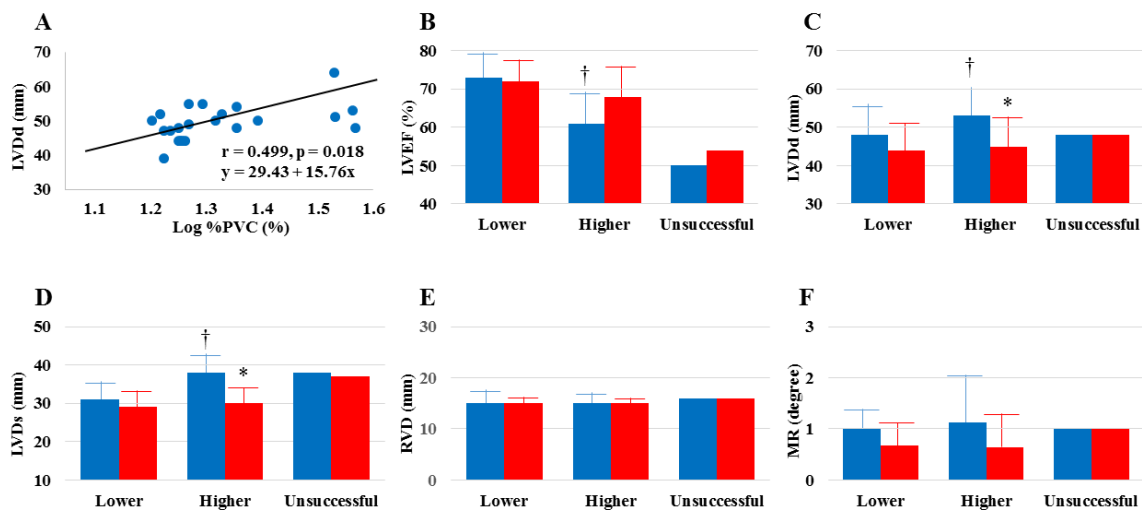

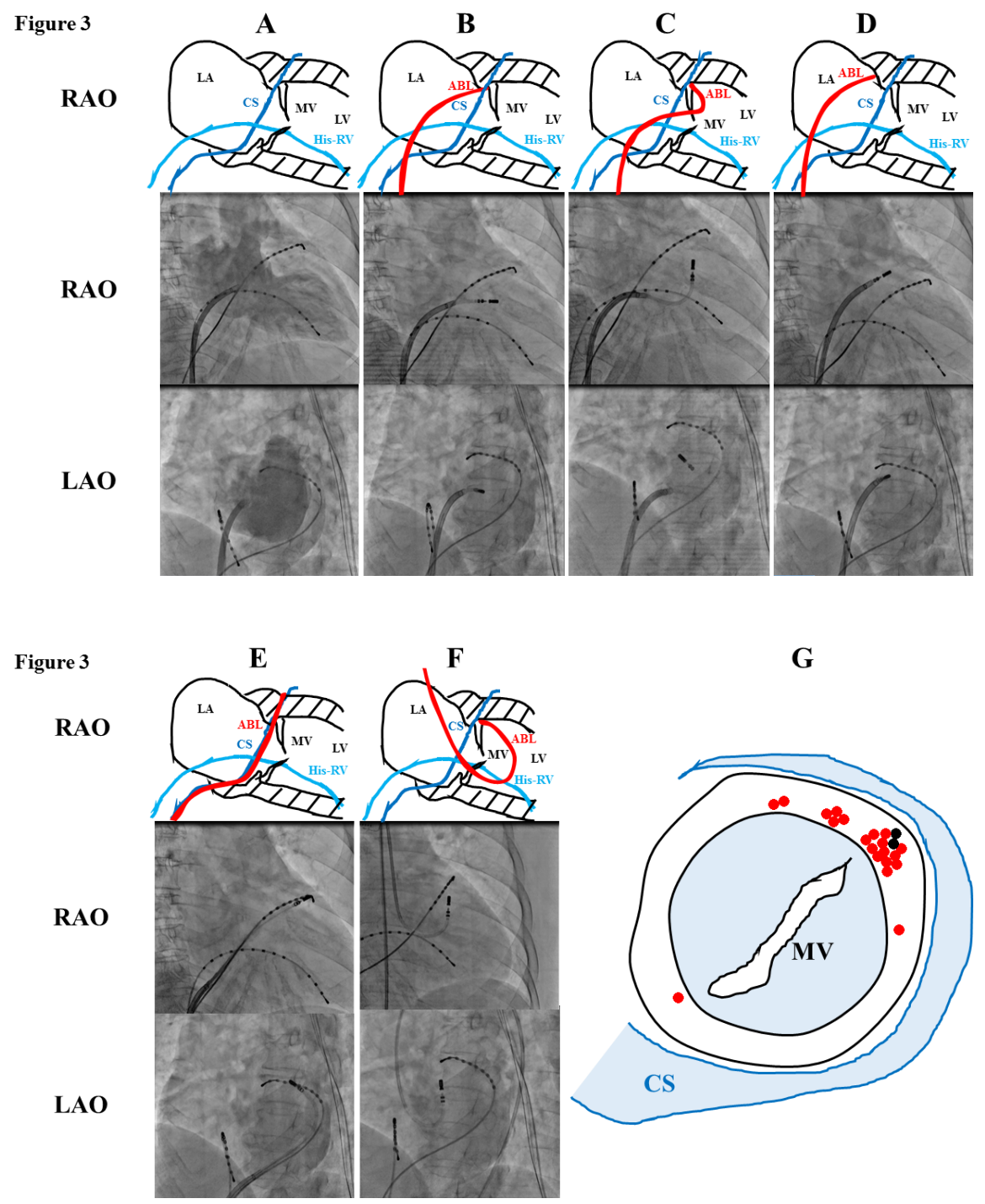